\title{
Intriguing parasites and intramembrane proteases
}

\author{
Robert B. Rawson ${ }^{1}$ \\ Department of Molecular Genetics, University of Texas Southwestern Medical Center, Dallas, Texas 75390, USA
}

\begin{abstract}
Rhomboid intramembrane proteases occur throughout the kingdoms of life. In this issue of Genes \& Development, Baxt and colleagues (pp. 1636-1646) report that the single proteolytic rhomboid (EhROM1) from Entamoeba histolytica cleaves cell surface galactose-binding or N-acetylgalactosamine-binding (Gal/Gal-NAc) lectins. EhROM1 and lectins colocalize during phagocytosis and receptor capping. EhROM1 is found at the base of the cap rather than in the cap proper, suggesting a role in receptor shedding and implying that EhROM1 is crucial for amoebal infection.
\end{abstract}

Parasitic protists are fascinating organisms in their own right, with their typically complex life cycles, distinctive physiologies, and uncertain relationships. They are of immense practical interest as well; parasitic infections account for an enormous proportion of the disease burden on the human population worldwide. The debilitating and often lethal parasitic diseases remain among the most intractable infections and result in between 1.5 and 3 million deaths annually. The enormous costs resulting from lost productivity and caring for the millions who suffer from parasitic disease represent significant hurdles to economic development throughout the world, and these burdens tend to be the highest where the resources to bear them are the least. Advances in our understanding of parasites, such as how they initiate and maintain infections, therefore, have significant potential to improve human health and well-being worldwide.

Among parasitic diseases, amoebiasis ranks second only to malaria in global morbidity, affecting vast numbers of people worldwide and killing some 100,000 annually (World Health Organization 1997). The causative agent of human amoebiasis, Entamoeba histolytica, faces many of the same challenges faced by other parasites when establishing infection. Baxt et al. (2008) detail the role of the single rhomboid intramembrane protease from $E$. histolytica in crucial parasite processes: phagocytosis and immune evasion.

[Keywords: Serine protease; presenilin; site-2 protease; erythrophagocytosis; immune evasion; pathogenesis]

${ }^{1}$ Correspondence.

E-MAIL Rob.Rawson@UTSouthwestern.Edu; FAX (214) 648-8804.

Article is online at http://www.genesdev.org/cgi/doi/10.1101/gad.1686808.

\section{The parasitic lifestyle}

Parasitism poses common challenges to the diverse organisms that practice it. Parasites must first locate and enter a host. Next, in many cases, host tissues or cells must be invaded, requiring some means of attachment and introgression. The successful parasite usually establishes a long-term infection, and this potentially affords the host ample time to mount a vigorous immune response to the invader. In order to survive and reproduce while "dining at another's table," the parasite must somehow manage to evade host immune defenses that may act at several stages of the infection cycle. Broadly, extracellular parasites are subject to the humoral response, while intracellular stages are the target of cellular immunity.

\section{Invasion and evasion}

Strategies for evading the host immune response are as complex and varied as the parasites that use them, and most parasites use several different strategies in the course of infection. Parasites may engage various responses to the host immune system (Zambrano-Villa et al. 2002). Some parasites synthesize proteases that specifically target components of the host immune machinery for destruction. Others send out molecules that mimic chemokines and suppress the immune response (Goodridge and Harnett 2005). In any event, modulating the host's immune response is a delicate matter. If a parasite disables the response sufficiently, competitors may then flourish in the immunocompromised host. Once the host succumbs, the parasite loses its meal ticket. Thus, strategies for dealing with the immune response are varied and subtle.

Many parasites have intricate life cycles involving multiple hosts and multiple developmental stages, each with its own characteristic array of antigenic proteins, even within a single host. The malarial parasite Plasmodium falciparum, for example, shuttles between intraand extracellular modes, moving from mosquito saliva to the bloodstream to hepatocytes and on to erythrocytes. At each stage, different antigens are elaborated (Kilejian 1980), and even within a single stage the dominant antigens can change, enabling the parasite to "hide" functionally from the host (Plebanski et al. 
1997) - by the time an effective response to one antigen is mounted, the parasite is presenting the immune system with a new one. If, after switching antigens, the parasite still faces immune challenge, it may simply shed the targeted antigens (Howell et al. 2003), much as a lizard may lose its tail to escape a menacing cat. E. histolytica uses a unique variation of this strategy, called capping (Fig. 1). The effectiveness of these various evasive strategies, alone or in combination, may account, in part, for the limited success of antiparasite vaccination strategies.

\section{Intramembrane proteolysis and parasitic infection}

Intramembrane proteolysis is an evolutionarily ancient signal transduction mechanism occurring widely in organisms ranging from prokaryotes to people (Brown et al. 2000). It was first uncovered a dozen years ago during efforts to understand diseases that are far more common in the aging populations of industrialized societies than are parasitic diseases: atherosclerosis and Alzheimer's disease.

\section{$\gamma$ Secretase}

Among the hallmarks of Alzheimer's disease are the socalled amyloid plaques that are associated with neurodegeneration. These plaques are formed largely of protein fragments from the amyloid precursor protein (APP). Release of these fragments, called amyloid $\beta$ peptide, requires two separate cleavages by two distinct enzymes: $\beta$ secretase and $\gamma$ secretase.

$\beta$ Secretase (or BACE) is an aspartyl protease. Its active site lies within a large domain that is disposed to the lumen of the secretory pathway. BACE is anchored to the membrane by a single membrane-spanning helix. $\gamma$ Secretase is a large complex composed of multiple polypeptide chains (Kimberly et al. 2003) including presenilin, which harbors the active site. The presenilins were originally identified by their genetic association with

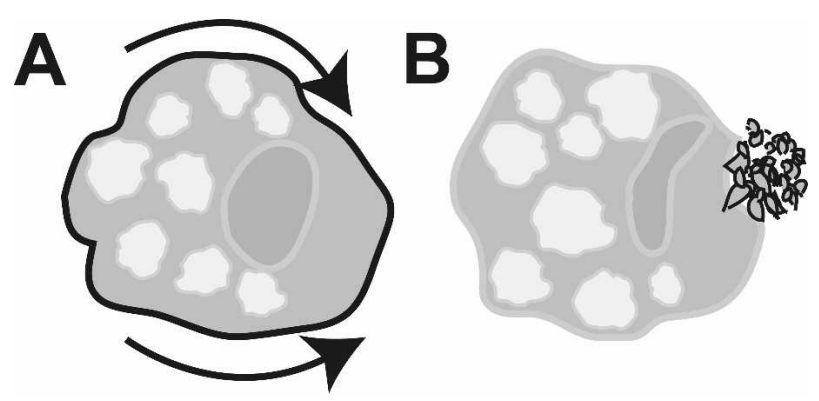

Figure 1. Surface receptor capping in Entamoeba histolytica. Cartoons show amoeba cells. $(A)$ Once antigens on the amoeba surface are recognized by host immune components (indicated by black line), they are rapidly translocated toward the posterior pole (arrows). (B) At the posterior end, membranes harboring surface receptors form into a complex array of vesicles termed the uroid prior to being shed (Silva et al. 1975). predisposition to early-onset Alzheimer's disease in certain families. They are aspartyl proteases comprised of multiple membrane-embedded helices.

Amyloid $\beta$ is released in two sequential steps: First, $\beta$ secretase cleaves APP within its luminal domain, near the surface of the membrane, and then $\gamma$ secretase cleaves it within its membrane-spanning helix (Selkoe 1996). In addition to APP, presenilins are required for the cleavage of other proteins such as Notch (Baumeister et al. 1997; De Strooper et al. 1999).

\section{Site-2 protease ( $S 2 P)$}

Excessively high levels of cholesterol in the bloodstream, hypercholesterolemia, leads to atherosclerosis, vascular disease, and death. Efforts to understand the global regulation of cholesterol metabolism in cells lead to the identification of the sterol regulatory elementbinding protein (SREBP) transcription factors (Brown and Goldstein 1999). SREBPs are made as precursors that reside initially in the ER membrane, owing to their two membrane-spanning helices. The transcriptionally active $\mathrm{N}$-terminal domain is released from the membrane by sequential cleavage of the precursor by two distinct proteases. The site-1 protease, a typical serine protease of the secretory pathway, anchored to the membrane by a single membrane-spanning helix, cleaves SREBPs in the short luminal sequence that separates its two membrane-spanning helices. The second cleavage occurs near the membrane surface, within the first membrane-spanning helix (Sakai et al. 1996; Duncan et al. 1998).

Cloning and sequencing the S2P cDNA revealed S2P to be a large, unusually hydrophobic metalloprotease unrelated to any previously described protein. Topological studies suggested that the active site was at or within the plane of the bilayer (Zelenski et al. 1999). This inference is supported by recent crystal structure analysis of a bacterial S2P homolog (Feng et al. 2007).

S2P was the first intramembrane-cleaving protease (or I-CliP) (Wolfe et al. 1999a) to be identified (Rawson et al. 1997). Subsequently, the presenilins were shown to be the $\gamma$ secretases (Wolfe et al. 1999b). These observations lead to the recognition of regulated intramembrane proteolysis as a widespread and ancient signal-transduction mechanism (Brown et al. 2000).

\section{Signal peptide peptidase (SPP)}

Studies of the fate of cleaved signal peptides lead to the identification of another family of aspartyl intramembrane proteases, the signal peptide peptidases (Weihofen et al. 2002). Outside of their conserved active sites, SPP and presenilin show no significant sequence similarity and SPP cleaves type II ( $\mathrm{N}$ terminus cytoplasmic) membrane-spanning helices rather than type I ( $\mathrm{N}$ terminus extracytoplasmic) helices (Dev et al. 2006).

S2P, the presenilins, and SPP share a requirement for prior cleavage of the substrate by a different protease before they can act. S2P substrates are the product of S1P 
cleavage (Rawson et al. 1997; Ye et al. 2000b), while APP needs to be cleaved by $\beta$ secretase before being cleaved by presenilins. Signal peptides are released from their precursor proteins by signal peptidase prior to cleavage by SPP (Weihofen et al. 2002).

\section{Rhomboid}

Analysis of Drosophila mutants harboring defects in epidermal growth factor (EGF) signaling revealed a fourth family of intramembrane cleaving proteases, the rhomboids (Urban et al. 2001). These were shown to be serine proteases. Unlike the other intramembrane proteases, rhomboids show no requirement for prior cleavage of the substrate.

In contrast to most other proteases, intramembranecleaving proteases evince little sequence specificity at or around the scissile bond. Some seem to require motifs distant from the site of cleavage (Hua et al. 1996; Lohi et al. 2004). For example, in cell-based assays of S2P activity, the residues flanking the scissile bond within the first membrane-spanning helix of SREBP could be substituted without affecting cleavage (Duncan et al. 1998), but a pair of asp-pro residues 11 residues distal to the site of cleavage proved crucial (Ye et al. 2000a). The asp-pro sequence, like other "helix-breaking" motifs, can form a boundary between the $\mathrm{N}$-terminal end of an $\alpha$ helix and more extended structures (Richardson and Richardson 1988).

Peptide bonds within an $\alpha$ helix are refractory to hydrolysis and the scissile bond must adopt an extended conformation in order to be cleaved. The helix-breaking residues probably serve to stabilize the partially unwound helices that are thought to be the substrates for intramembranous cleavage (Ye et al. 2000a). In common with other I-Clips such as S2P and SPP, rhomboids also require helix-breaking motifs within their substrates (Lemberg and Martoglio 2002; Urban and Freeman 2003). Potential substrates for I-CLiPs may be identified by searching data bases for predicted membrane-spanning helices that harbor these helix-breaking motifs. Using this approach, for example, several proteins from the parasite Toxoplasma gondii were identified, tested, and shown to be substrates of rhomboid proteases (Urban and Freeman 2003).

T. gondii is an apicomplexan and an obligate intracellular parasite. The apicomplexans use active mechanisms to invade host cells. In contrast to other intracellular parasites, these mechanisms do not rely on the host endocytic machinery. Invasion requires T. gondii to bind to host cell receptors with high affinity. The parasite then reorients itself so that its apical end is disposed to the host cell plasma membrane. Interactions between the parasite cytoskeleton and the cytoplasmic domain of its host protein-binding proteins enable apicomplexans to force themselves into their hosts. Interactions between the host and parasite must then be undone in order to complete entry and form the distinctive parasitophorous vacuole.

One way these parasites undo interactions with host surface proteins is through intramembrane proteolysis of the interacting proteins (Opitz et al. 2002; Zhou et al. 2004). Additional studies identified the Toxoplasma rhomboids required for key cleavage events during host cell invasion and intracellular growth (Zhou et al. 2004; Brossier et al. 2005, 2008). Similar rhomboids have been found in all other apicomplexans for which genomic sequences are available, including Cryptosporidium and Plasmodium species (Dowse and Soldati 2005), where they appear to play similar roles (O'Donnell et al. 2006; Singh et al. 2007). Most parasites do not use active entry into host cells, however, and the role of rhomboids in other parasites has remained unclear.

\section{Entamoeba histolytica}

In contrast to the baroque life cycles pursued by many parasites, that of E. histolytica seems prosaically uncomplicated. This protist passes from human host to human host by the simple expedient of fecal-to-oral transmission of tetranucleate cysts. These enter the host through contaminated food or water. Once in the lumen of the gut, excystation and division produce eight trophozoites from each cyst. The trophozoites migrate to the large intestine, where they attach to the mucosa and multiply by binary fission. Most remain attached to the mucosa, where they feed on intestinal bacteria. At this stage, no symptoms of disease are present. Some trophozoites undergo encystation and are excreted in the feces, thus perpetuating the cycle.

Occasionally, some trophozoites invade the intestinal mucosa, establishing a symptomatic infection accompanied by the cellular lysis for which the species is aptly named. This results in characteristic flask-shaped lesions within the enteric tissue. In a few cases, the amoebae progress through host tissues and into the bloodstream, enabling them to invade other organs (principally the liver, but also the brain and lungs) and form potentially lethal amebic abscesses (Haque et al. 2003).

At each of these stages, the invader is subject to attack by the immune system: principally IgG in tissues and blood, IgM in the gut. To avoid the consequences of having its surface antigens recognized by immunoglobulins, E. histolytica uses a strategy of actively sequestering opsonized surface antigens to its posterior pole, in an area of enfolded membrane called the uroid (Fig. 1). Once there, the antigens are shed. This presumably allows the amoeba to go about its business unmolested. Transport of surface proteins to the uroid is reminiscent of "capping" in lymphocytes and was first described for E. histolytica more than 30 years ago (Da Silva and MartinezPalomo 1974; Silva et al. 1975).

Owing to its apparent importance in allowing the amoeba to survive in an immunocompetent host, the process of capping and uroid formation has been vigorously studied. Polarized movement of surface antigens involves actin and myosin (Arhets et al. 1995); agents that disrupt the cytoskeleton block capping and uroid formation (Espinosa-Cantellano and Martinez-Palomo 1994). Cytoskeletal involvement in the polarized trans- 
port of opsonized surface proteins is clear; the mechanisms responsible for shedding these antigens are less well characterized.

\section{Amoebal proteases}

The genome E. histolytica encodes at least 45 proteases, three-quarters of which are cysteine proteases (Loftus et al. 2005). Some amoebal proteases are intracellular and others are found on the cell surface, while yet others are secreted into the surroundings. The expression of proteases correlates with virulence (Gadasi and Kessler 1983; Keene et al. 1990; Hirata et al. 2007), owing, in part, to their role in degrading the intestinal mucus barrier (Moncada et al. 2005, 2006; Lidell et al. 2006). Amoebal proteases are also involved in contact-dependent lysis of host cells (Singh et al. 2004) and in the degradation of host immune factors (Herdman et al. 1997; Tran et al. 1998). Thus, amoebal proteases are well-established contributors to virulence and disease.

The genome of E. histolytica encodes a single rhomboid protein harboring the residues necessary for catalysis, EhROM1 (Baxt et al. 2008) This was unexpected, since most eukaryotic genomes encode multiple catalytically competent rhomboids. Biochemical analyses of EhROM1 activity reveal that it differs from most other rhomboids in not being able to cleave Spitz, the first rhomboid substrate identified. Instead, EhROM1 can cleave adhesin molecules from $P$. falciparum. These substrates have small helix-relaxing residues within the $\mathrm{N}$ terminal half of their membrane-spanning helices, preceded by large aromatic residues, features that render them refractory to cleavage by most other rhomboid proteases. Thus, EhROM1 shares similar specificity with a $P$. falciparum rhomboid, PfROM4, that cleaves the adhesins during host cell invasion (Baker et al. 2006). A search of the E. histolytica genome for substrates harboring the noncanonical motif turned up five genes encoding the large subunit of the Gal/GalNAc lectin. This lectin is important during development, invasion of host tissues, and evasion of the immune response (Petri et al. 2002).

Intriguingly, in two seemingly unrelated processes, phagocytosis and surface receptor capping, EhROM1 and Gal/Gal-NAc lectin colocalize. Most proteins associated with capping have been found to accumulate within the uroid itself, presumably as cargo. EhROM1, on the other hand, accumulates in a cup shape at the base of the uroid. This suggests that rather than being cargo, the amoebal rhomboid plays an active role in the formation or the release of the uroid and its associated surface receptors.

\section{Conclusion}

Examples of intramembrane proteolysis are known from all kingdoms of life. Although originally recognized as a widespread mechanism of signal transduction, recent findings expand its roles to other cellular functions. In apicomplexans like T. gondii and P. falciparum, rhomboids participate in the active invasion of host cells by cleaving adhesins and are thus essential for these obligate intracellular parasites (Brossier et al. 2005, 2008; Baker et al. 2006).

The current report by Baxt et al. (2008) implicates $E$. histolytica EhROM1 in phagocytosis and surface receptor shedding during immune evasion. EhROM1 is therefore expected to be an essential gene in this protozoan. As such, the single rhomboid from E. histolytica may be an attractive, selective target for antiamoebal chemotherapy. Moreover, identification of roles for EhROM1 and PfROM4 in the cleavage of adhesion proteins indicates that this role for intramembrane proteolysis predates the divergence of amoebas and apicomplexans. It may be that the original I-CliPs played similar roles before putative gene duplication events permitted their cooption into signaling pathways. The findings of Baxt et al. (2008) also suggest a productive avenue for the investigation of the other eukaryotic rhomboids whose functions are currently unknown.

\section{Acknowledgments}

Thanks to Kaori Tanaka and Krista Matthews for helpful comments. Work in the Rawson laboratory is supported by the National Institutes of Health and the Perot Family Foundation.

\section{References}

Arhets, P., Gounon, P., Sansonetti, P., and Guillen, N. 1995. Myosin II is involved in capping and uroid formation in the human pathogen Entamoeba histolytica. Infect. Immun. 63: 4358-4367.

Baker, R.P., Wijetilaka, R., and Urban, S. 2006. Two Plasmodium rhomboid proteases preferentially cleave different adhesins implicated in all invasive stages of malaria. PLoS Pathog. 2: e113. doi: 10.1371/journal.ppat.0020113.

Baumeister, R., Leimer, U., Zweckbronner, I., Jakubek, C., Grunberg, J., and Haass, C. 1997. Human presenilin-1, but not familial Alzheimer's disease (FAD) mutants, facilitate Caenorhabditis elegans Notch signalling independently of proteolytic processing. Genes Funct. 1: 149-159.

Baxt, L.A., Baker, R.P., Singh, U., and Urban, S. 2008. An Entamoeba histolytica rhomboid protease with atypical specificity cleaves a surface lectin involved in phagocytosis and immune evasion. Genes \& Dev. (this issue), doi: 10.1101/ gad.1667708.

Brossier, F., Jewett, T.J., Sibley, L.D., and Urban, S. 2005. A spatially localized rhomboid protease cleaves cell surface adhesins essential for invasion by Toxoplasma. Proc. Natl. Acad. Sci. 102: 4146-4151.

Brossier, F., Starnes, G.L., Beatty, W.L., and Sibley, L.D. 2008. Microneme rhomboid protease TgROM1 is required for efficient intracellular growth of Toxoplasma gondii. Eukaryot. Cell 7: 664-674.

Brown, M.S. and Goldstein, J.L. 1999. A proteolytic pathway that controls the cholesterol content of membranes, cells, and blood. Proc. Natl. Acad. Sci. 96: 11041-11048.

Brown, M.S., Ye, J., Rawson, R.B., and Goldstein, J.L. 2000. Regulated intramembrane proteolysis: A control mechanism conserved from bacteria to humans. Cell 100: 391-398. 
Da Silva, P.P. and Martinez-Palomo, A. 1974. Induced redistribution of membrane particles, anionic sites and con A receptors in Entamoeba histolytica. Nature 249: 170-171.

De Strooper, B., Annaert, W., Cupers, P., Saftig, P., Craessaerts, K., Mumm, J.S., Schroeter, E.H., Schrijvers, V., Wolfe, M.S., Ray, W.J., et al. 1999. A presenilin-1-dependent $\gamma$-secretaselike protease mediates release of Notch intracellular domain. Nature 398: 518-522.

Dev, K.K., Chatterjee, S., Osinde, M., Stauffer, D., Morgan, H., Kobialko, M., Dengler, U., Rueeger, H., Martoglio, B., and Rovelli, G. 2006. Signal peptide peptidase dependent cleavage of type II transmembrane substrates releases intracellular and extracellular signals. Eur. J. Pharmacol. 540: 10-17.

Dowse, T.J. and Soldati, D. 2005. Rhomboid-like proteins in Apicomplexa: Phylogeny and nomenclature. Trends Parasitol. 21: 254-258.

Duncan, E.A., Dave, U.P., Sakai, J., Goldstein, J.L., and Brown, M.S. 1998. Second-site cleavage in sterol regulatory elementbinding protein occurs at transmembrane junction as determined by cysteine panning. J. Biol. Chem. 273: 1780117809.

Espinosa-Cantellano, M. and Martinez-Palomo, A. 1994. Entamoeba histolytica: Mechanism of surface receptor capping. Exp. Parasitol. 79: 424-435.

Feng, L., Yan, H., Wu, Z., Yan, N., Wang, Z., Jeffrey, P.D., and Shi, Y. 2007. Structure of a site-2 protease family intramembrane metalloprotease. Science 318: 1608-1612.

Gadasi, H. and Kessler, E. 1983. Correlation of virulence and collagenolytic activity in Entamoeba histolytica. Infect. Immun. 39: 528-531.

Goodridge, H.S. and Harnett, M.M. 2005. Introduction to immune cell signalling. Parasitology 130 (Suppl.): S3-S9.

Haque, R., Huston, C.D., Hughes, M., Houpt, E., and Petri Jr., W.A. 2003. Amebiasis. N. Engl. J. Med. 348: 1565-1573.

Herdman, D.S., Tran, V., Torian, B., and Reed, S.L. 1997. Cleavage of IgG by the neutral cysteine proteinase of E. histolytica. Arch. Med. Res. 28 Spec. No: 178-179.

Hirata, K.K., Que, X., Melendez-Lopez, S.G., Debnath, A., Myers, S., Herdman, D.S., Orozco, E., Bhattacharya, A., McKerrow, J.H., and Reed, S.L. 2007. A phagocytosis mutant of Entamoeba histolytica is less virulent due to deficient proteinase expression and release. Exp. Parasitol. 115: 192-199.

Howell, S.A., Well, I., Fleck, S.L., Kettleborough, C., Collins, C.R., and Blackman, M.J. 2003. A single malaria merozoite serine protease mediates shedding of multiple surface proteins by juxtamembrane cleavage. J. Biol. Chem. 278: 2389023898.

Hua, X., Sakai, J., Brown, M.S., and Goldstein, J.L. 1996. Regulated cleavage of sterol regulatory element binding proteins requires sequences on both sides of the endoplasmic reticulum membrane. J. Biol. Chem. 271: 10379-10384.

Keene, W.E., Hidalgo, M.E., Orozco, E., and McKerrow, J.H. 1990. Entamoeba histolytica: Correlation of the cytopathic effect of virulent trophozoites with secretion of a cysteine proteinase. Exp. Parasitol. 71: 199-206.

Kilejian, A. 1980. Stage-specific proteins and glycoproteins of Plasmodium falciparum: Identification of antigens unique to schizonts and merozoites. Proc. Natl. Acad. Sci. 77: 36953699.

Kimberly, W., LaVoie, M.J., Ostaszewski, B.L., Ye, W., Wolfe, M.S., and Selkoe, D.J. 2003. $\gamma$-Secretase is a membrane protein complex comprised of presenilin, nicastrin, Aph-1, and Pen-2. Proc. Natl. Acad. Sci. 100: 6382-6387.

Lemberg, M.K. and Martoglio, B. 2002. Requirements for signal peptide peptidase-catalyzed intramembrane proteolysis. Mol. Cell 10: 735-744.
Lidell, M.E., Moncada, D.M., Chadee, K., and Hansson, G.C. 2006. Entamoeba histolytica cysteine proteases cleave the MUC2 mucin in its C-terminal domain and dissolve the protective colonic mucus gel. Proc. Natl. Acad. Sci. 103: 9298-9303.

Loftus, B., Anderson, I., Davies, R., Alsmark, U.C., Samuelson, J., Amedeo, P., Roncaglia, P., Berriman, M., Hirt, R.P., Mann, B.J., et al. 2005. The genome of the protist parasite Entamoeba histolytica. Nature 433: 865-868.

Lohi, O., Urban, S., and Freeman, M. 2004. Diverse substrate recognition mechanisms for rhomboids; thrombomodulin is cleaved by Mammalian rhomboids. Curr. Biol. 14: 236-241.

Moncada, D., Keller, K., and Chadee, K. 2005. Entamoeba histolytica-secreted products degrade colonic mucin oligosaccharides. Infect. Immun. 73: 3790-3793.

Moncada, D., Keller, K., Ankri, S., Mirelman, D., and Chadee, K. 2006. Antisense inhibition of Entamoeba histolytica cysteine proteases inhibits colonic mucus degradation. Gastroenterology 130: 721-730.

O'Donnell, R.A., Hackett, F., Howell, S.A., Treeck, M., Struck, N., Krnajski, Z., Withers-Martinez, C., Gilberger, T.W., and Blackman, M.J. 2006. Intramembrane proteolysis mediates shedding of a key adhesin during erythrocyte invasion by the malaria parasite. J. Cell Biol. 174: 1023-1033.

Opitz, C., Di Cristina, M., Reiss, M., Ruppert, T., Crisanti, A., and Soldati, D. 2002. Intramembrane cleavage of microneme proteins at the surface of the apicomplexan parasite Toxoplasma gondii. EMBO J. 21: 1577-1585.

Petri Jr., W.A., Haque, R., and Mann, B.J. 2002. The bittersweet interface of parasite and host: Lectin-carbohydrate interactions during human invasion by the parasite Entamoeba histolytica. Annu. Rev. Microbiol. 56: 39-64.

Plebanski, M., Lee, E.A., and Hill, A.V. 1997. Immune evasion in malaria: Altered peptide ligands of the circumsporozoite protein. Parasitology 115 (Suppl.): S55-S66.

Rawson, R.B., Zelenski, N.G., Nijhawan, D., Ye, J., Sakai, J., Hasan, M.T., Chang, T.Y., Brown, M.S., and Goldstein, J.L. 1997. Complementation cloning of S2P, a gene encoding a putative metalloprotease required for intramembrane cleavage of SREBPs. Mol. Cell 1: 47-57.

Richardson, J.S. and Richardson, D.C. 1988. Amino acid preferences for specific locations at the ends of $\alpha$ helices. Science 240: $1648-1652$.

Sakai, J., Duncan, E.A., Rawson, R.B., Hua, X., Brown, M.S., and Goldstein, J.L. 1996. Sterol-regulated release of SREBP-2 from cell membranes requires two sequential cleavages, one within a transmembrane segment. Cell 85: 1037-1046.

Selkoe, D.J. 1996. Amyloid $\beta$-protein and the genetics of Alzheimer's disease. J. Biol. Chem. 271: 18295-18298.

Silva, P.P., Martinez-Palomo, A., and Gonzalez-Robles, A. 1975. Membrane structure and surface coat of Entamoeba histolytica. Topochemistry and dynamics of the cell surface: Cap formation and microexudate. J. Cell Biol. 64: 538-550.

Singh, D., Naik, S.R., and Naik, S. 2004. Role of cysteine proteinase of Entamoeba histolytica in target cell death. Parasitology 129: 127-135.

Singh, S., Plassmeyer, M., Gaur, D., and Miller, L.H. 2007. Mononeme: A new secretory organelle in Plasmodium falciparum merozoites identified by localization of rhomboid-1 protease. Proc. Nat1. Acad. Sci. 104: 20043-20048.

Tran, V.Q., Herdman, D.S., Torian, B.E., and Reed, S.L. 1998. The neutral cysteine proteinase of Entamoeba histolytica degrades IgG and prevents its binding. J. Infect. Dis. 177: 508-511.

Urban, S. and Freeman, M. 2003. Substrate specificity of rhomboid intramembrane proteases is governed by helix-breaking 
residues in the substrate transmembrane domain. Mol. Cell 11: $1425-1434$.

Urban, S., Lee, J.R., and Freeman, M. 2001. Drosophila rhomboid-1 defines a family of putative intramembrane serine proteases. Cell 107: 173-182.

Weihofen, A., Binns, K., Lemberg, M.K., Ashman, K., and Martoglio, B. 2002. Identification of signal peptide peptidase, a presenilin-type aspartic protease. Science 296: 2215-2218.

Wolfe, M.S., De Los Angeles, J., Miller, D.D., Xia, W., and Selkoe, D.J. 1999a. Are presenilins intramembrane-cleaving proteases? Implications for the molecular mechanism of Alzheimer's disease. Biochemistry 38: 11223-11230.

Wolfe, M.S., Xia, W., Ostaszewski, B.L., Diehl, T.S., Kimberly, W.T., and Selkoe, D.J. 1999b. Two transmembrane aspartates in presenilin-1 required for presenilin endoproteolysis and gamma-secretase activity. Nature 398: 513-517.

World Health Organization 1997. Entamoeba taxonomy. Bull. World Health Organ. 75: 291-294.

Ye, J., Dave, U.P., Grishin, N.V., Goldstein, J.L., and Brown, M.S. 2000a. Asparagine-proline sequence within membranespanning segment of SREBP triggers intramembrane cleavage by site-2 protease. Proc. Nat1. Acad. Sci. 97: 5123-5128.

Ye, J., Rawson, R.B., Komuro, R., Chen, X., Dave, U.P., Prywes, R., Brown, M.S., and Goldstein, J.L. 2000b. ER stress induces cleavage of membrane-bound ATF6 by the same proteases that process SREBPs. Mol. Cell 6: 1355-1364.

Zambrano-Villa, S., Rosales-Borjas, D., Carrero, J.C., and OrtizOrtiz, L. 2002. How protozoan parasites evade the immune response. Trends Parasitol. 18: 272-278.

Zelenski, N.G., Rawson, R.B., Brown, M.S., and Goldstein, J.L. 1999. Membrane topology of S2P, a protein required for intramembranous cleavage of sterol regulatory element-binding proteins. J. Biol. Chem. 274: 21973-21980.

Zhou, X.W., Blackman, M.J., Howell, S.A., and Carruthers, V.B. 2004. Proteomic analysis of cleavage events reveals a dynamic two-step mechanism for proteolysis of a key parasite adhesive complex. Mol. Cell. Proteomics 3: 565-576. 


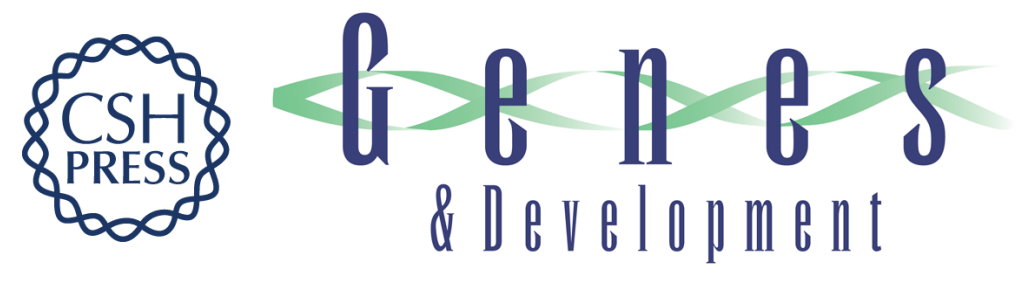

\title{
Intriguing parasites and intramembrane proteases
}

\author{
Robert B. Rawson
}

Genes Dev. 2008, 22:

Access the most recent version at doi:10.1101/gad.1686808

\section{Related Content An Entamoeba histolytica rhomboid protease with atypical specificity cleaves a surface lectin involved in phagocytosis and immune evasion Leigh A. Baxt, Rosanna P. Baker, Upinder Singh, et al. \\ Genes Dev. June , 2008 22: 1636-1646}

References This article cites 54 articles, 24 of which can be accessed free at: http://genesdev.cshlp.org/content/22/12/1561.full.html\#ref-list-1

Articles cited in:

http://genesdev.cshlp.org/content/22/12/1561.full.html\#related-urls

\section{License}

Email Alerting Receive free email alerts when new articles cite this article - sign up in the box at the top Service right corner of the article or click here.

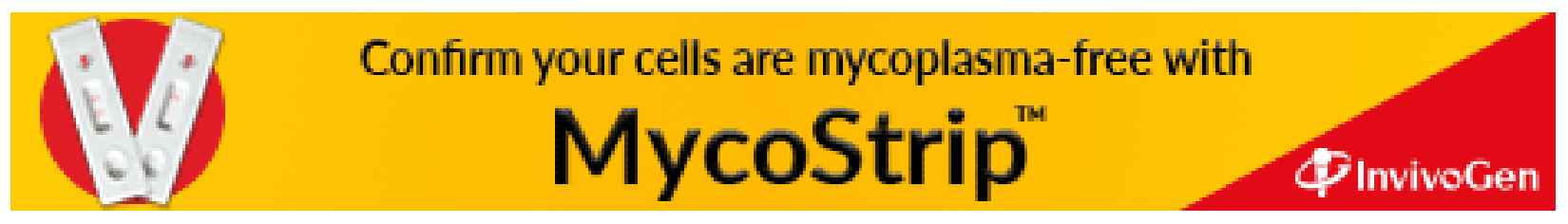

